\title{
Enunciación
}

\section{Aproximación a los géneros académicos y profesionales usados en el ámbito jurídico}

\author{
Approach to academic and professional genres used in legal field \\ David Alberto Londoño Vásauez* \\ Álvaro Ramírez Botero**
}

\section{Resumen}

Este trabajo hace una aproximación a los géneros académicos y profesionales del ámbito jurídico que se evidencian en una población de docentes y egresados del programa de Derecho de la Institución Universitaria de Envigado. El artículo es producto de una investigación cualitativa, hermenéutica-comprensiva, a partir del análisis de los resultados de la aplicación de encuestas semiestructuradas que contenían preguntas cerradas y abiertas. Los resultados mostraron que para los docentes los géneros más usados en la enseñanza son los textos doctrinales, jurisprudenciales, demandas, códigos y tutelas, mientras que para los egresados los textos que más usan en su ejercicio profesional son los de normativa, jurisprudencia y leyes. Esto permite concluir que existe un alto grado de coincidencia entre los géneros jurídicos usados en la formación y los del ejercicio profesional, a lo que se puede sumar que son géneros discursivos que, en la práctica profesional, forman parte del tecnolecto y en el ejercicio profesional se conocen, se aplican y pueden ser objeto de ejercicio crítico.

Palabras clave: géneros discursivos, géneros profesionales, géneros jurídicos, tecnolecto legal.

\begin{abstract}
This paper provides an approach to the academic and professional genres of the legal field that are evident in a population of teachers and graduates of the law program of the Envigado University Institution. The paper is the product of a qualitative, hermeneutic-comprehensive research, based on the analysis of the results of the application of semi-structured surveys that contained closed and open questions. The results showed that, for teachers, the most used genres in teaching are doctrinal, jurisprudential, demands, codes and guardianship texts, while for graduates, the texts that they use the most in their professional practice, are those of regulations, jurisprudence, and laws. This allows concluding that there is a high degree of coincidence between the legal genres used in training and those of professional practice, to which it can be added that they are discursive genres that, in professional practice in addition to being part of the professional jargon and in professional practice, they are known, applied and can be critically exercised.
\end{abstract}

Keywords: discursive genres, professional genres, legal genres, legal jargon.

* Doctor en Ciencias Sociales, Niñez y Juventud, miembro del grupo de investigación en Psicología Aplicada y Sociedad, de la Institución Universitaria de Envigado. Docente de tiempo completo de la Institución Universitaria de Envigado. Correo electrónico: dalondono@correo.iue.edu.co

** Doctor en Humanidades, director del grupo de investigación Psicología Aplicada y Sociedad, de la Institución Universitaria de Envigado. Decano de la Facultad de Ciencias Sociales de la Institución Universitaria de Envigado. Correo electrónico: alvaro.ramirez@iue.edu.co

Cómo citar: Londoño Vásquez, D. A. y Ramírez Botero, A. (2020). Aproximación a los géneros académicos y profesionales usados en el ámbito jurídico. Enunciación, 25(2), 191-205. https://doi.org/10.14483/22486798.16131

Artículo recibido: 7 de abril de 2020; aprobado: 12 de junio de 2020 


\section{Introducción}

Los niveles de lectura y escritura en los procesos de formación académica han venido ganando mayor representatividad frente a la relación que tienen en el aprendizaje de los saberes disciplinares (Carlino, 2005; Castelló, 2009; Torres, 2018). La comprensión en estos contextos específicos requiere no solo del manejo del código lingüístico y el conocimiento de la superestructura textual, sino también de los conceptos precisos expresados en términos, los cuales conforman los diferentes tipos de textos que se trabajan en la formación, proponiendo una relación sociedad/conocimiento/individuo (Castañeda y Henao, 2005; Parodi, Ibáñez, Venegas y González, 2010; Fuster, 2016).

Lo anterior tiene relevancia para el análisis de las prácticas letradas disciplinares (López, 2002; Izquierdo, 2006; Orlando, 2014) del ámbito jurídico (Dejarnatt, 2001; García y Agüero, 2014; Aguilar, 2017). Esto, porque los futuros abogados, probablemente, aprenden tanto lo conceptual como lo textual a partir de la exposición a los diferentes textos estudiados en su formación, presentados o dialogados por y con sus docentes, los cuales son fortalecidos por su funcionalidad y uso en el mundo laboral (Inglehart, Narko y Zimmerman, 2003; Betancurt y Frías, 2015; Londoño, Ramírez y Garay, 2019).

Algunas investigaciones han indagado sobre los géneros académicos y profesionales, relacionados con la variación disciplinar como las realizadas por Daniel Cassany y Carmen López (2010); María Castro, Laura Hernández y Martín Sánchez (2010); Estrella Montolío y Anna López (2010); Giovanni Parodi, Romualdo Ibáñez, Rene Venegas y Cristian González (2010); Paulina Meza (2015), y David Londoño, Álvaro Ramírez y Kennier Garay (2019). No obstante, no hay un consenso frente a qué es un género, cómo se tipifica y cuáles son sus funciones cognitivas, sociales y culturales.

El género, inicialmente, se entendió como un objeto de interés de las comunidades discursivas, cuyo propósito comunicativo difiere e identifica al género per se. Por tanto, cada género cuenta con una serie de actos comunicativos que comparten unos propósitos y estos se convierten en prototipos dentro de la comunidad discursiva (Swales, 1990); es decir, cada propósito comunicativo dentro de dicha comunidad requiere de una forma para hacerlo.

No obstante, el género puede ser entendido como el fruto de una práctica social en el contexto generado por grupos humanos (Bajtín, 1982), eso es, un artefacto textual convencionalizado o institucionalizado en contextos de prácticas disciplinarias que permiten concebir cómo miembros de comunidades discursivas específicas construyen, interpretan y usan estos artefactos (orales o escritos) para alcanzar los propósitos comunicativos (Bhatia, 2002). De allí que sea pertinente tener presente los propósitos (objetivos de la comunidad institucionalizada y sus intereses comunicativos), los productos (artefactos textuales o géneros), las prácticas (discursivas, procedimientos y procesos) y los participantes (miembros de una comunidad discursiva) (Bhatia, 1999).

Por consiguiente, se podría afirmar que el género está estrechamente relacionado con las variopintas formas abstractas y sociales de emplear el lenguaje dentro de un contexto. Así, se presupone que los textos dependen del dónde y del para qué fueron creados, puesto que es a través de las formas convencionalizadas de comunicación que los individuos construyen relaciones académicas, laborales, profesionales, entre otras (Hyland, 2003). Esto permite que los géneros discursivos sean considerados dinámicos, situados, organizados en forma y contenido, lo que permite la construcción y reproducción de estructuras sociales a partir de la delimitación de comunidades discursivas desde lo cognitivo, lo interpersonal, lo social y lo gremial (Cassany, 2006).

En otras palabras, se podría entender el género como una construcción social, cultural y cognitiva que responde a necesidades comunicativas propias 
de un grupo de individuos, cuyos propósitos pueden variar dependiendo de los contextos (académicos, profesionales o disciplinares). De allí, la importancia que adquieren los géneros en la formación de conocimientos especializados (Parodi, 2008), especialmente, en las universidades, cuando algunos de los géneros estudiados en la formación de pregrado no son necesariamente los requeridos en el ámbito profesional. Esto, pues se espera que el estudiante de pregrado en su formación, a partir del uso de ciertos géneros, pueda irse introduciendo como miembro de la respectiva comunidad discursiva en pro de apropiarse de la comprensión y producción de las diferentes prácticas comunicativas características de dicha comunidad.

Por tanto, los géneros académicos y profesionales han venido ganando un reconocimiento en el proceso de formación disciplinario. De allí que, cada vez, sea más recurrente que las universidades centren su atención en los niveles de comprensión lectora (Schwarts, 2008; Schugar, Schugar y Penny, 2011; Gordillo y Flórez, 2017) y de producción escritural (Schumacher, 2006; Cassany y Morales, 2009; Legarre y Vitetta, 2016) de sus estudiantes desde una perspectiva de género textual (Bhatia, 2002; Ruiz y Camps, 2009; Aguilar, 2017), donde se tienen presente el lenguaje especializado (AIbi, 2007; Tiersma, Tiersa y Solan, 2012; Kittredge y Lehrberger, 2015), el discurso académico (Nelken, 2004; Henao, Londoño, Frías y Marín, 2010; Wingate, 2012) o profesional (López, 2002; Montolío y López, 2010; Torres, 2018), dependiendo de la perspectiva asumida.

Dentro de la tradición formativa de los abogados, parece que los niveles de comprensión y producción textual de los estudiantes se afianzan a través de la interacción con sus docentes (Londoño, Ramírez y Garay, 2019). Se repite constantemente que "la mejor forma de enseñar a los estudiantes a pensar como abogados es entrenarlos para escribir, hablar y actuar como tal" (Schumacher, 2006, p. 103). En otras palabras, el proceso de formación que predomina en el campo del Derecho parece responder a la capacidad que tiene el docente para presentar las temáticas correspondientes a su curso, sobrepasando la transmisión de información y centrándose en la construcción discursiva disciplinaria (DeJarnatt, 2001), la cual podría obedecer a cómo se han adquirido los conocimientos y cómo se vehicula el acceso al saber; es decir, aspectos de modalidad epistémica y de evidencialidad (López, 2002), propios del discurso legal: lenguaje oral y escrito en ámbitos jurídicos y del derecho (Kurzon, 1997), esto es lenguaje jurídico (Ríos, 2005).

Por ello, para Aguilar (2017), las necesidades a las cuales debe enfrentarse un estudiante de Derecho en su proceso de formación, son "comprender el código de comunicación de los abogados, conocer los distintos contextos en los que se produce la comunicación especializada y ser capaz de manipular los géneros especializados para responder a situaciones nuevas e inesperadas son necesidades" (p. 184); además de las dificultades inherentes en la comprensión y la escritura jurídica como producto de su compleja terminología en dicha disciplina (Bhatia, 2008).

Aquí, la selección de los géneros que se van a usar en los procesos de formación puede no ser lo suficientemente significativa para los estudiantes de Derecho, puesto que, en algunas ocasiones, los géneros académicos empleados por los docentes apuntan a otros propósitos comunicativos no profesionalizantes (Parodi, Boudon y Julio, 2015) y, de hecho, hasta por fuera de la misma disciplina del derecho (Betancurt y Frías, 2015). Sin embargo, es claro que los denominados géneros jurídicos se usan para lograr la apropiación del tecnolecto (Albi, 2007). Por otra parte, en relación con los géneros jurídicos, Alcaraz y Hughes (2002) mencionan que los más comunes son: "títulos y diplomas, certificados, leyes, repertorios de jurisprudencia, sentencias, escrituras, contratos, pólizas de seguro, testamentos, poderes notariales y artículos profesionales" (p. 101).

Por otro lado, Aguilar (2017), referente a los géneros discursivos escritos y de ambiente 
universitario jurídico en Chile, propone tres clasificaciones. La primera es la de géneros relativos a la escritura como destreza profesional. Esta está compuesta por informe en Derecho (amicus curiae), escrito forense (demanda, contestación, apelación), resolución judicial (auto, decreto, sentencia, interlocutoria, sentencia definitiva) y contratos (de compra venta, de arriendo y de trabajo). La segunda es la de géneros relativos a procesos de investigación jurídica. Aquí se subdividen en textos de nivel inicial (textos analíticos, informe de lectura, ensayo argumentativo) e investigación jurídica formativa (tesis, tesina y proyecto de investigación). Finalmente, la tercera clasificación, la de género de conocimiento jurídico se conforma de la doctrina jurídica (libro, artículo, reseña, comentario, jurisprudencia) y los reportes no jurídicos (artículo de prensa, reportaje, editorial y entrevista).

Finalmente, Londoño, Ramírez y Garay (2019), en una investigación sobre prácticas de literacidad en dos facultades de Derecho en Colombia, encontraron los siguientes géneros jurídicos predominantes en el proceso de formación: el acto y auto jurídico, el alegato, el auto judicial, el contrato, la demanda, el dictamen, el fallo, la intervención, el memorial, la resolución, la sentencia y la tutela. Estos permitieron la construcción de categorías de géneros doctrinales, jurisprudenciales, normativos, ético-filosóficos y de literatura jurídica. Cabe mencionar que la selección de los géneros jurídicos usados en la enseñanza por los docentes se realiza de forma presuntiva y siguiendo elementos canónicos y tradicionales del curso a cargo.

Teniendo en cuenta lo anterior, resulta importante identificar los géneros académicos y profesionales usados en el ámbito jurídico en una Facultad de Ciencias Políticas y Jurídicas de Envigado (Colombia) en pro de afianzar los procesos de comprensión y producción textual presentes en la formación de los abogados, estableciendo puntos de encuentro entre los géneros jurídicos y su funcionalidad en la formación.

\section{Metodología}

La investigación realizada fue cualitativa (Wingate, 2012; Silverman, 2016), hermenéutica-comprensiva (Herrera, 2010; Londoño y Castañeda, 2010). Esto permitió la identificación de los géneros académicos y profesionales, como se mencionó anteriormente, a partir de la participación de los agentes involucrados.

Por tanto, los participantes fueron diez docentes de un programa de Derecho de una institución universitaria pública de Envigado (Colombia), uno por semestre académico, responsable de un curso en el 2019-I, y los egresados de dicho programa que se encontraban matriculados en uno de los posgrados de la Facultad de Ciencias Políticas y Jurídicas de dicha institución para el semestre 2019II (36 egresados en total).

En relación con los docentes, estos fueron seleccionados por la Decanatura de la Facultad, de forma intencional (Scharager y Reyes, 2001; Martínez, 2012). Las condiciones proporcionadas por los investigadores a la decanatura para dicha selección fueron: docentes vinculados al programa, uno por semestre académico (de allí que fueran diez participantes) y que estuvieran en disposición de participar y diligenciar el consentimiento informado.

Los docentes seleccionados correspondían a los siguientes cursos: Técnicas Comunicativas I y II (TCl y TCII), Dogmática Constitucional (DC), Teoría General del Proceso (TGP), Administrativo General (AG), Laboral Colectivo (LC), Proceso Laboral $(\mathrm{PL})$, Métodos y Solución de Conflictos (MSC), Responsabilidad del Estado (RE) y Jurisprudencia (J), mencionados en orden desde el primer al décimo semestre.

La técnica empleada fue la encuesta semiestructurada (Martínez, 2012; Páramo, 2018). Se diseñaron dos encuestas. La primera para los docentes. Esta se dividió en dos partes: información sociocultural como la edad, la formación de pregrado y posgrado, años de experiencia profesional, 
docencia y docencia en la Facultad y en preguntas relacionadas con los usos de textos en sus clases, los tipos de textos usados, los criterios para su selección y la relación que estos tienen con los posibles textos que debe usar un abogado en su ejercicio. Con respecto a la segunda, para los egresados, se dividió igualmente en dos partes: información sociocultural y prácticas de comprensión y lectura en su proceso de formación como abogados y en su práctica profesional.

Para la elaboración de ambas encuestas y sus respectivas rúbricas, se contó con la asesoría de un profesional en Derecho, con formación de Maestría y 15 años de experiencia en el litigio, y en el proceso de validación (Henao et al., 2010; Bol, Sáiz y Pérez, 2013), se contó con la revisión de tres investigadores expertos de Colombia, Argentina y Chile, en el área de lingüística, géneros discursivos y escritura académica, los tres con formación doctoral.

Con respecto al diligenciamiento de las encuestas, los docentes lo realizaron en el Laboratorio de Psicología de la Facultad de Ciencias Sociales de dicha institución, lugar propicio por su diseño y posibilidad de agrupar a los investigadores con los participantes. Estos se demoraron entre 20 y 40 minutos para diligenciar la encuesta. Las instrucciones fueron dadas al comienzo del diligenciamiento por uno de los investigadores. Estas se centraban en el nombre de la investigación y el interés por conocer la perspectiva de los docentes frente a los géneros discursivos y académicos, en pro de evitar cualquier direccionamiento en las respuestas.

Por otro lado, los investigadores visitaron los grupos correspondientes a cinco programas de posgrados ofertados por la Facultad para 2019-II, en el transcurso de tres semanas. Allí encuestaron a los asistentes que desearon participar y estuvieron de acuerdo con diligenciar el consentimiento informado. Previamente, los investigadores habían acordado solo seleccionar las encuestas diligenciadas por los egresados del programa de Derecho de la institución, que tuvieran un año o más de experiencia laboral. Por tanto, de 43 encuestas realizadas, solo se analizaron 36 .

Finalmente, el análisis de la información recolectada en las encuestas a docentes y egresados fue realizado a través de las rúbricas y sistematizado con una matriz de análisis cualitativo (Cassani y Morales, 2009; Cardona, Valencia, Duque y Londoño, 2015). A continuación, se presentan los resultados con su respectivo análisis.

\section{Resultados y análisis}

En este apartado se presenta de forma discriminada el análisis realizado tanto a las encuestas diligenciadas por los docentes como por los egresados.

\section{Análisis de la encuesta realizada a do- centes/participantes del programa de Derecho}

En lo concerniente a la información sociocultural, se encontró que predomina el género masculino (80\%) frente al femenino (20\%) en los docentes/ participantes, aspecto que parece ser una tendencia en el resto del programa. Los rangos de edades predominantes son de 31 a 40 años (con 5 participantes) y de 41 a 50 años (con 5 participantes), como se evidencia en la figura 1.

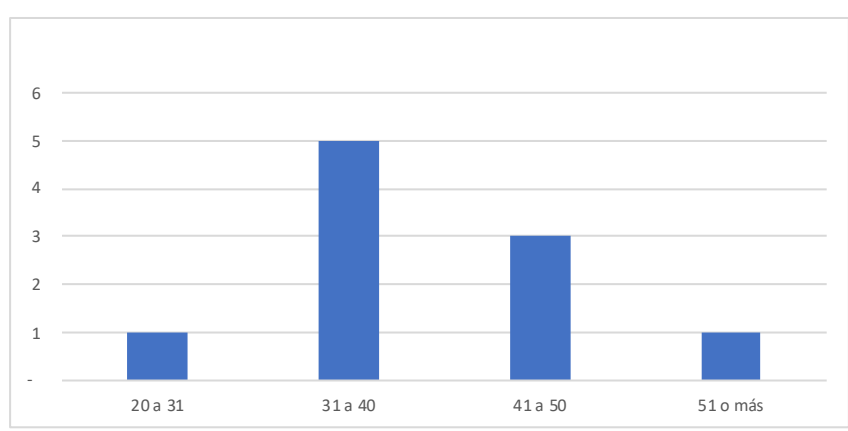

Figura 1. Agrupación de rango de edades de los docentes participantes

Fuente: elaboración propia. 
De igual forma, los pregrados predominantes son Derecho (80\%) y Filosofía (20\%), especialmente, por la selección de los cursos, pues desde el tercer al décimo semestre, corresponden a cursos de fundamentación disciplinar y del área profesional. No obstante, resulta interesante que los dos cursos de Técnicas Comunicativas I y II estén a cargo de profesores con pregrado en Filosofía, quienes además son los dos únicos docentes con formación doctoral, de los participantes (doctor en Ciencias Políticas y doctor en Filosofía) los demás docentes cuentan con formación posgradual entre especialización y maestría. Sin representar esto variaciones significativas referidas a los géneros usados, excepto en los cursos de técnicas comunicativas, que no son disciplinares, pero también usan géneros del ámbito jurídico.

La experiencia profesional es igual o mayor a la docente y, a su vez, en docencia en dicha Facultad. Los promedios en años de experiencia profesional son de 10,4, en comparación de 8,9 en docencia y 8,4 en la facultad. Con respecto a rangos de agrupación, predominan de 1 a 5 años con cuatro participantes (solo una docente participante cuenta con un año de experiencia tanto profesional como en docencia) y de 11 a 15 años con otros 4 participantes, como se observa en la figura 2.

En cuanto a la segunda parte de la encuesta, esta se compone de 11 preguntas abiertas. En aras de facilitar el análisis de esta parte narrativa, se

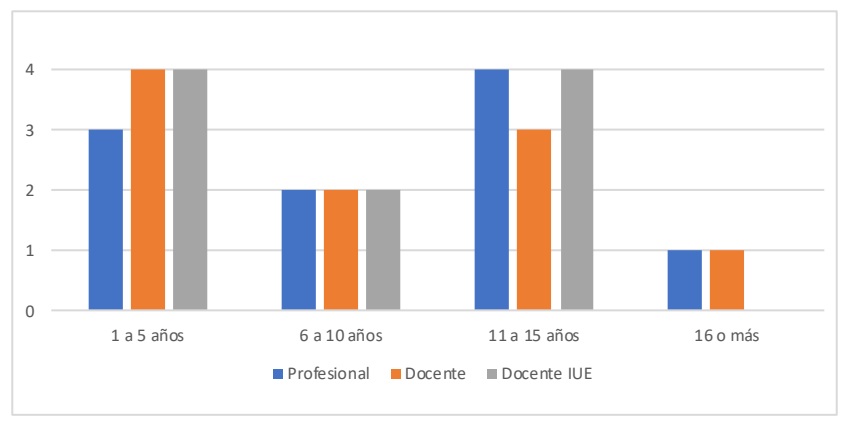

Figura 2. Agrupación de experiencia profesional, docencia y en la facultad

Fuente: elaboración propia. sistematizaron por preguntas y las 10 respuestas obtenidas.

La primera pregunta fue: ¿Cómo concibe la lectura y la escritura en el proceso de formación de los estudiantes de Derecho? Los 10 participantes están de acuerdo sobre el rol de la lectura y la escritura en el proceso de formación en Derecho, a tal punto que se repiten adjetivos como fundamental, esencial, clave, determinante, importante y necesaria.

Herramienta esencial para la formación de un proceso crítico y reflexivo. (TCl, 24/07/2019)

Absolutamente fundamentales ambas, un abogado bien sea litigante, docente o investigador tendrá como herramientas principales la escritura y la lectura. (TGP, 24/07/2019)

Determinante, pues los profesionales del Derecho deben contar con una competencia del más alto nivel es este ámbito para poder no solo obtener su título, sino además para ejercer la profesión con responsabilidad social. (LC, 24/07/2019) ${ }^{1}$

Además, conciben la lectura y la escritura como una herramienta y como componentes necesarios para acceder a la información específica de sus respectivos cursos, la cual se espera que pueda convertirse en procesos comprensivos que permitan la generación de conocimiento y afianzamiento del tecnolecto.

Son dos componentes no reductibles a la mera formación en Derecho, pero sí son condiciones necesarias y no suficientes para una formación integral. (TCII, 24/07/2019)

La lectura es la clave para ahondar en el conocimiento y con la escritura es que debe apropiarse el mismo, máxime que se maneja en la rama judicial el tema de las demandas por escrito, al igual que las diferentes teorías de los casos; y para de igual manera escribir sobre lo que se hace a nivel académico. (AG, 24/07/2019)

1 Se utilizan las siglas de las asignaturas de los docentes seleccionados para la elaboración de las encuestas. Estas asignaturas fueron enunciadas en la metodología. 
Es básica ya que deben formarse intelectualmente para poder expresar adecuada y pertinentemente sus conceptos. (MSC, 24/07/2019)

De igual forma, dos de los participantes plantean utilizar ambas habilidades como una opción pedagógica que permita la formación de abogados más competentes:

La lectura y la escritura son importantes en el desarrollo de competencias básicas. Como estrategia pedagógica y herramienta didáctica, la lectura y la escritura que se haga con sentido y significado fortalece el desarrollo de los futuros profesionales. (PL, 24/07/2019)

Para un estudiante de Derecho, la lectura y la escritura es fundamental. Por tanto, es menester emplearlos para crear excelentes procesos de enseñanza, que, a su vez, facilitan el aprendizaje; más allá de una memoria formal y repetitiva; con una mirada crítica y pragmática. (J, 24/07/2019)

Por tanto, se puede evidenciar que la lectura y la escritura gozan de un reconocimiento metacognitivo por parte de los docentes encuestados, los cuales entienden el papel de estas dos habilidades en la formación profesional de sus estudiantes como vital, especialmente, teniendo en cuenta que, tradicionalmente, la lectura ha sido una herramienta didáctica recurrente en las prácticas pedagógicas de formación en Derecho. Lo anterior, debido a la lectura de la norma y la explicación jurisprudencial o doctrinal en clase.

Con respecto a la segunda y tercera pregunta - ¿Realiza actividades de escritura en sus cursos?, y ¿qué actividades realiza en su curso en base en la lectura y la escritura?-, la totalidad de los participantes afirman que realizan actividades de escritura en sus cursos. Aspecto relevante si se tiene presente el valor asignado en el proceso de formación del abogado. No obstante, parece que las actividades de lectura y escritura que realizan responden más a un interés de cada docente que a un requerimiento específico del microcurrículo o del programa como tal. Algunas de las actividades mencionadas son:

Lectura: oratoria, discurso, homilía, cuento, expresión oral y escrita, audiencia, poesía, discurso académico y político. Escritura: ensayo, cuento, dictado, escritura libre, técnicas de redacción, estrella, cubo, mapas de ideas, conceptual, mental, falacias argumentativas, torbellino de ideas, poesía, discurso académico y político. (TCl, 24/07/2019)

Escritura de ensayos breves, ejercicios de lectura crítica (tipo Saber Pro en modo virtual) y discusiones críticas en clase. (TCII, 24/07/2019)

Que lean diferentes sentencias y textos, y que hagan ensayos y demandas. (AG, 24/07/2019)

Es decir, se enfatiza en la importancia de la lectura y la escritura, y estas aparecen en los cursos, pero no responden a un diseño programático o al cumplimiento de objetivos de formación, sino a una aplicación de estas como medio de acceso a información y de evaluación respondiendo a requerimientos del Ministerio de Educación Nacional (MEN) de Colombia, a través de sus pruebas censales.

No obstante, en los cursos de fundamentación disciplinar y profesional, es más recurrente la lectura de textos disciplinares como normas, códigos, sentencias, demandas y contestaciones.

Lectura: encargo el estudio sentencial de las altas cortes, el estudio de textos de doctrina jurídica. (LC, 24/07/2019)

Lectura de normas tales como el Código Sustantivo de Trabajo y el Código de Procedimientos Laboral, en donde se le de aplicación a la norma. (PL, 24/07/2019)

En mis cursos se realizan actividades de lecturas de sentencias de los Altos Tribunales: Corte Constitucional, Corte Suprema de Justicia y Consejo del Estado. Estas lecturas están orientadas a desencriptar las razones de fondo que sustentan los fallos judiciales. (J, 24/07/2019) 
Pero en pocas ocasiones se menciona el recurso de las actividades escriturales y, cuando se hace, se relaciona más con informes de lectura que con la escritura de alguno de los géneros discursivos disciplinares propios del curso o del Derecho.

Revisión de jurisprudencia, lectura de artículos, redacción de informes de lectura. (DC, 24/07/2019)

Escritura: no encargo amplias tareas; a veces elaboración de un informe de una audiencia. (LC, 24/07/2019)

Así entonces propongo a los estudiantes lecturas de sentencias y ellos elaboran informes escritos, de forma principal, con su aporte personal. (RE, $24 / 07 / 2019)$

En otras palabras, la lectura está más consolidada en las prácticas de los docentes encuestados, especialmente, en los cursos de fundamentación disciplinar o profesional, pero, probablemente, por el material de estudio de base, más que por propósitos académicos de formación relacionados con los géneros discursivos predominantes en los cursos.

Por otro lado, la escritura se ubica en actividades compositivas o reflexivas que permiten al docente evidenciar el cumplimiento de una tarea comprensiva y los recursos más recurrentes son aquellos géneros académicos como el ensayo o una reflexión, el primero como uno de los géneros más solicitados en la universidad (González y Vega, 2010) y, el informe de lectura.

Las preguntas cuarta y quinta indagan sobre el número de textos leídos y escritos en el curso por los estudiantes. De los 10 participantes, 3 afirman que sus estudiantes, en sus respectivos cursos, leen 10, 16 y 20 (Responsabilidad del Estado, Jurisprudencia y Técnicas Comunicativas I, respectivamente) documentos en el semestre aproximadamente. Aspecto inquietante porque el promedio obtenido de los otros 7 participantes es de 3,8 textos por semestre. En lo concerniente a la escritura, el promedio disminuye a 2,5 entre 9 de los participantes, puesto que el docente de Técnicas Comunicativas I indica que sus estudiantes escriben alrededor de 20 textos en el semestre.

La sexta pregunta fue: ¿Trabaja otro tipo de textos que no sean escritos? Solo los docentes de Dogmática Constitucional y Jurisprudencia contestaron que no. Los otros participantes incluyen videos, imágenes, memes, mapas conceptuales, películas, audios de audiencias y conferencias.

En las siguientes preguntas - ¿Cuáles son los tres textos más importantes que usa en su curso?, y ¿cuál es el campo de aplicación de los textos?-, las respuestas a los tres textos más importantes variaron considerablemente, respondiendo a la característica de cada curso y de la temática allí trabajada. No obstante, la pregunta sobre los campos de aplicación permitió evidenciar cuáles eran los más predominantes. Los resultados indican que los textos más importantes en los cursos a cargo de los participantes encuestados pertenecen al campo doctrinal (44\%), seguido de jurisprudencial (25\%), normativo (17\%) y ético-filosófico (14\%). Este último referenciado, principalmente, por los docentes de Técnicas Comunicativas I y II, quienes cuentan con formación de pregrado en Filosofía, como se mencionó anteriormente.

La novena pregunta se centra en los criterios de selección de los textos para los cursos en mención. Aquí aparece una lista de criterios como textos cánones (relevancia), claridad conceptual (pertinencia) y nueva jurisprudencia y normativa (actualidad).

A partir de la claridad conceptual con que se recogen las categorías de la dogmática constitucional. (DC, 24/07/2019)

Reconocimiento del autor. Relación con la temática de clase. Disponibilidad del material. (TGP, 24/07/2019)

[...] actualidad y coherencia con el ordenamiento jurídico colombiano. (LC, 24/07/2019)

Además, de forma recurrente se menciona el microcurrículo del curso como una fuente inicial de consulta a la hora de seleccionar 
los textos; de allí, lo significativo de los colectivos docentes y la coordinación académica en la constante y oportuna revisión de este instrumento pedagógico-administrativo.

Finalmente, las dos últimas preguntas fueron: ¿Cuáles son los tipos de textos de derecho escritos más usuales en el ejercicio profesional del abogado?, y ¿cuáles de los textos que deben elaborar los estudiantes en sus clases cree que permiten desarrollar habilidades para el ejercicio profesional del Derecho? La lista obtenida, en relación con los textos escritos más usuales en el ejercicio, es amplia y varía considerablemente dependiendo del participante. Sin embargo, aquellos que tuvieron 3 o más recurrencias fueron: textos doctrinales (5), jurisprudenciales (4), demandas (3), códigos (3) y tutelas (3). Por otro lado, los textos trabajados en clase que permiten desarrollar habilidades para el ejercicio profesional del Derecho con 3 o más recurrencias fueron: demandas (4), contestación (4) y sentencia (3).

\section{Análisis de encuesta aplicada a egresados del programa de Derecho}

Los egresados, según los criterios previamente descritos en la metodología, diligenciaron las encuestas en espacios proporcionados por la Decanatura y la Coordinación de las Especializaciones, durante el desarrollo de sus actividades académicas, esto permitió el acompañamiento y la superación de dudas referidas al instrumento. En total fueron 36 encuestas de egresados distribuidas en los diferentes programas de especialización (tabla 1).

Tabla 1. Encuestas válidas por programa de posgrado

\begin{tabular}{lc}
\hline $\begin{array}{c}\text { Programa de posgrado en } \\
\text { Derecho }\end{array}$ & $\begin{array}{c}\text { Número de } \\
\text { encuestas válidas }\end{array}$ \\
\hline Contratación estatal & 10 \\
\hline Derecho administrativo laboral & 16 \\
\hline Derecho disciplinario & 7 \\
\hline Responsabilidad estatal & 3 \\
\hline
\end{tabular}

Fuente: elaboración propia.
En lo referido a la información sociodemográfica, se encontró que el 55,6 \% son mujeres, mientras que el 44,4 \% son hombres. Esta proporción es contraria a lo que se presenta en los docentes encuestados, donde el $80 \%$ son hombres y el $20 \%$ mujeres.

Por otra parte, en la información referida a la edad se encontró que el $50 \%$ de los encuestados están entre los 31 y 40 años, seguidos por el $30 \%$ entre los 20 y los 31 años, como se observa en la figura 3.

Frente a la experiencia profesional de los egresados que fueron encuestados, se encuentra dispersión, aunque hay un predominio del rango que va entre uno y dos años, mientras que solo hay tres egresados con experiencia entre 10 y 12 años (figura 4).

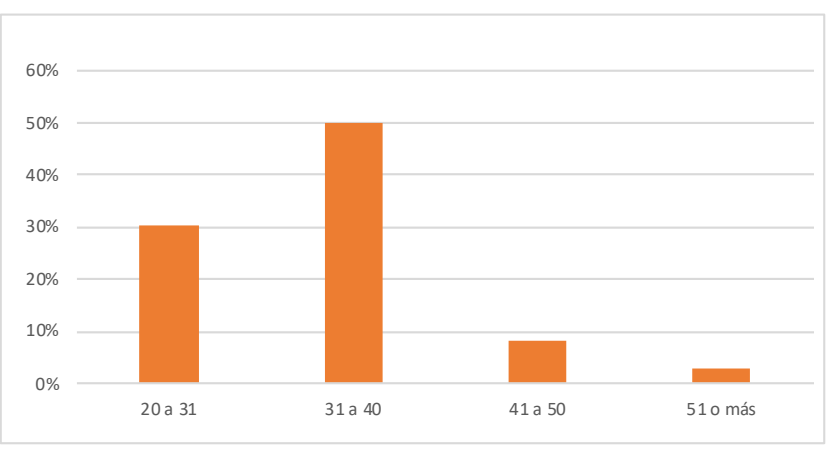

Figura 3. Agrupación de rango de edades de egresados encuestado

Fuente: elaboración propia.

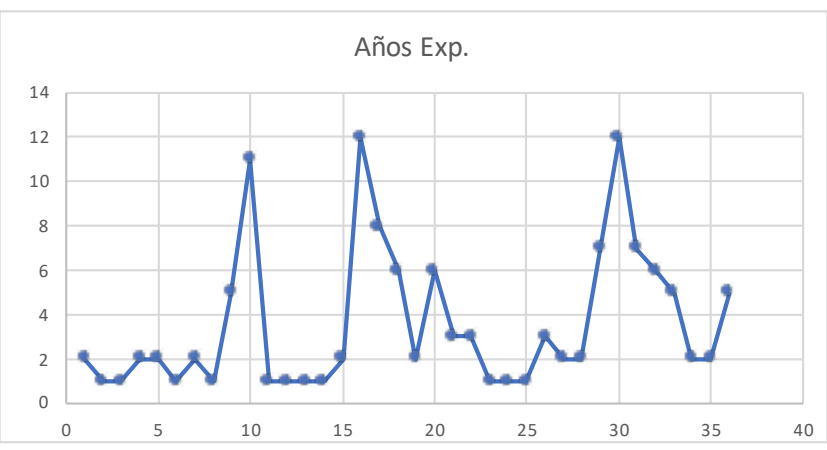

Figura 4. Años de experiencia de los egresados encuestados

Fuente: elaboración propia. 
Por otro lado, en la encuesta se indagó por las áreas del Derecho en las cuales se desempeñaban como profesionales, esto contando con que solo en pocas ocasiones laboran en una sola área. De esta forma se obtuvo que los egresados encuestados que están cursando los programas de pregrado laboran en las áreas del derecho detalladas en la figura 5.

De acuerdo con esto, se puede plantear que, principalmente, laboran en las áreas de derecho administrativo y derecho civil, seguido de derecho de familia y laboral.

En la segunda parte de la encuesta se indagó por los aspectos relacionados con la presencia de la lectura y la escritura en su ejercicio profesional. Los resultados obtenidos fueron:

En primer lugar, frente a la importancia que le dan a la lectura y la escritura en el ejercicio profesional se encontró que el $96 \%$ de los egresados la valoran con 5 , y el $4 \%$, con 4 , es decir que le conceden gran importancia. Posteriormente se indagó por el tipo de textos que leen y escribe en su ejercicio profesional, se obtuvo lo ilustrado en la figura 6.

En cuanto a los textos que leen, se observa que hay una dispersión significativa pero no un predominio significativo de algún tipo de texto. El texto que mayor lectura presenta son las leyes, asunto que es apenas lógico en el ejercicio

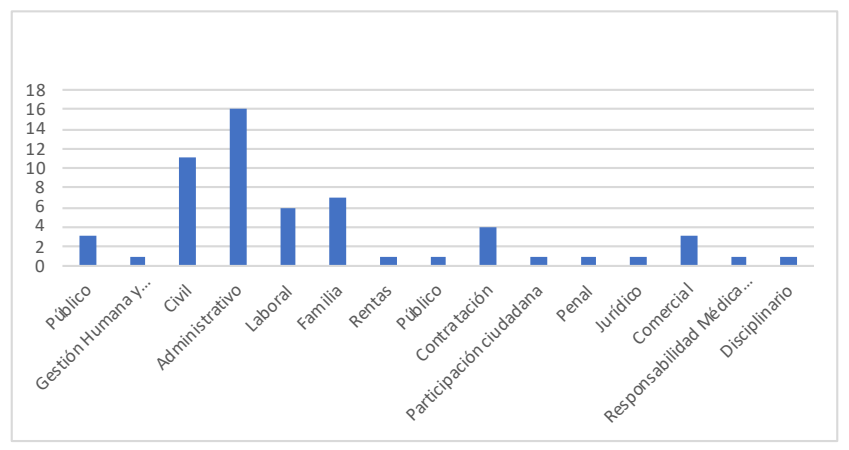

Figura 5. Áreas del derecho en que laboran los egresados encuestados

Fuente: elaboración propia. profesional, pues su labor se desarrolla a partir de las estimaciones de ley.

Ahora, frente a los textos que escriben se obtuvo lo ilustrado en la figura 7.

De acuerdo con esto, los textos que más escriben son tutelas, derechos de petición, demandas y contestaciones. Esto tiene una relación directa con las áreas de trabajo de los egresados que, aunque presenta variedad, hay una concentración en derecho civil, administrativo, laboral y familia.

$\mathrm{Al}$ hacer un comparativo entre los textos que leen y los textos que escriben, en el ejercicio profesional se obtuvo lo detallado en la figura 8.

Existe cierto grado de coincidencia entre los textos que leen y escriben, como las tutelas, los derechos de petición, las demandas, las contestaciones.

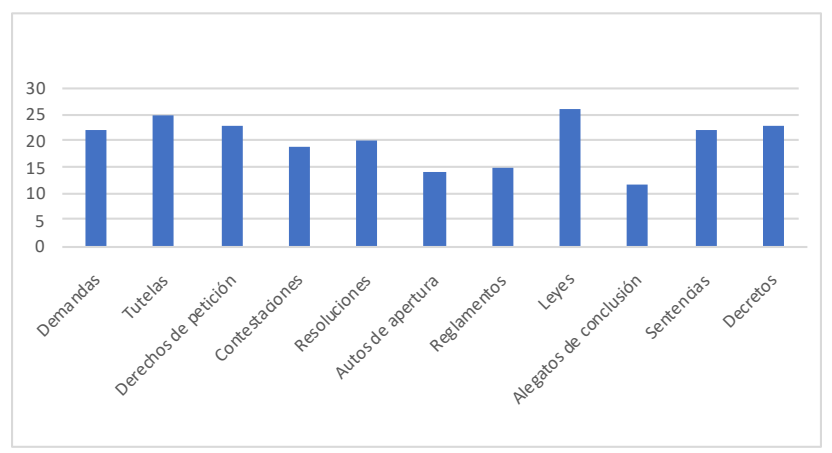

Figura 6. Textos que leen en el ejercicio profesional

Fuente: elaboración propia.

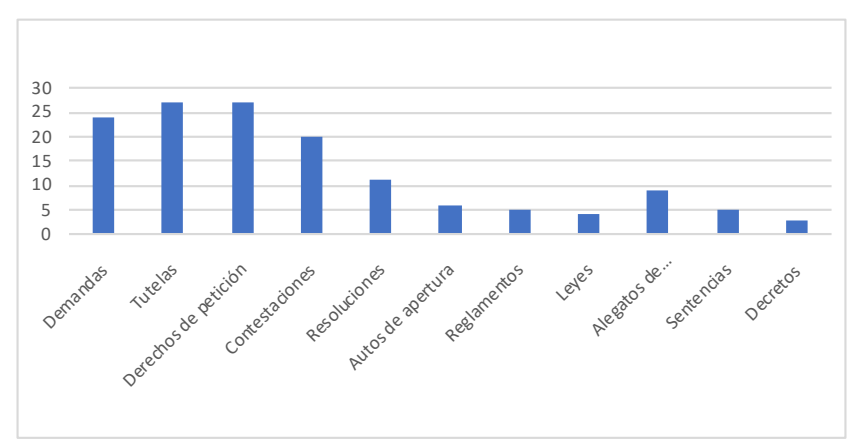

Figura 7. Textos que escriben en el ejercicio profesional

Fuente: elaboración propia. 
Esto tiene sentido en la medida en que son los tipos de textos que constantemente vinculan al ciudadano común y corriente, y a las organizaciones con la institucionalidad.

Sin embargo, se nota también la diferencia que existe entre la lectura y la escritura de textos como leyes, sentencias, decretos y reglamentos. El sentido de este hallazgo está en el ejercicio laboral de los abogados, puesto que una mínima parte de ellos redactan este tipo de textos, que están ligados solo a ciertos roles profesionales y cargos específicos.

Por otro lado, al indagar sobre la relación entre los textos que leen y escriben como profesionales, y los que leyeron y escribieron durante su formación, se encontró lo ilustrado en la figura 9.

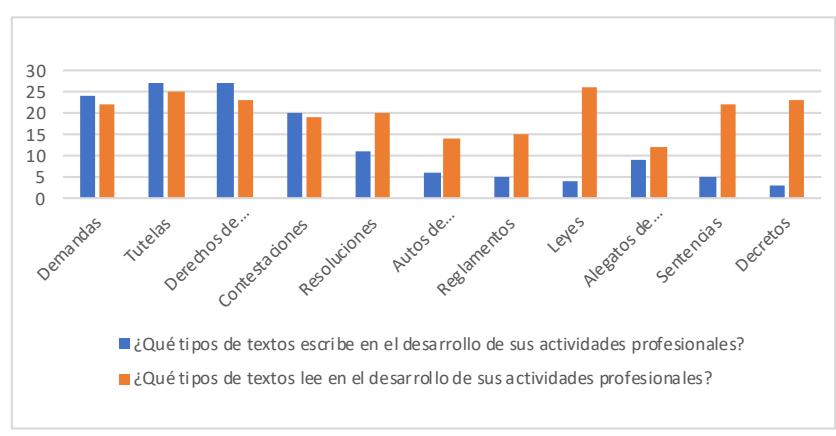

Figura 8. Comparativo de textos que leen y textos que escriben en el ejercicio profesional

Fuente: elaboración propia.

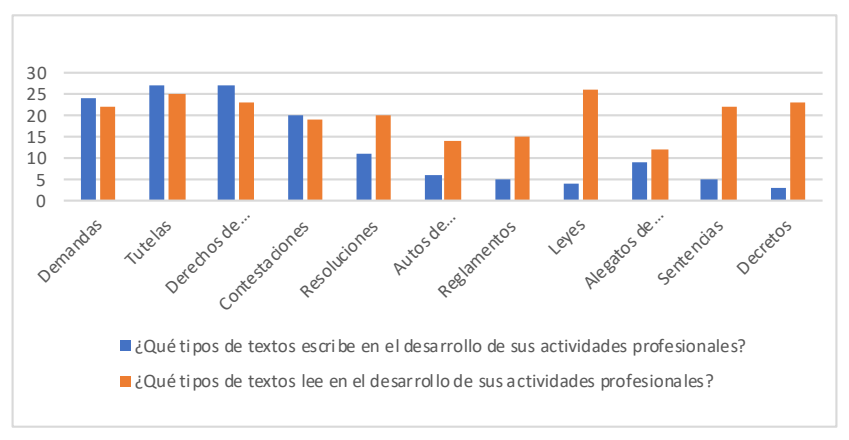

Figura 9. Comparativo textos que leen y que escriben en el ejercicio profesional y los que leyeron y escribieron durante su formación

Fuente: elaboración propia.
Se observa que los textos que coinciden son: demandas, tutelas, derechos de petición, resoluciones, leyes, sentencias y decretos. De esta forma, se puede plantear que hay coincidencia, y que al menos los mencionados que se trabajan en la formación están presentes durante el ejercicio profesional. Estas coincidencias se constatan al indagar específicamente por los textos de la formación que coinciden con el ejercicio profesional (figura 10).

Esto permite asegurar que los textos que más usan en su ejercicio profesional son los de normativa, seguido por jurisprudencia, textos referidos a las leyes, que se constituyen en textos rectores del ejercicio profesional de derecho.

\section{Conclusiones}

Los docentes de Derecho reiteran la importancia que tienen en sus clases la lectura y la escritura, pero no mencionan prácticas pedagógicas intencionales frente a estos. Sin embargo, la selección de los textos para trabajar en clase pareciera responder a dos posibles intenciones: una ilustrativa, de cómo es cada uno de los géneros más predominantes ligados, a su parecer, al hacer del abogado, y la otra propende por la ampliación de los conceptos jurídicos, normativos y legales que

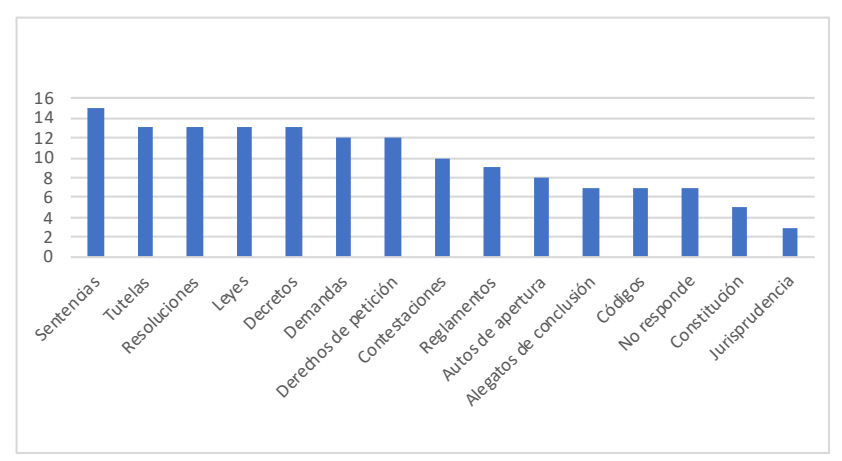

Figura 10. Textos de la formación que coinciden con los usados en el ejercicio profesional

Fuente: elaboración propia. 
refuerzan los contenidos temáticos de los cursos, los cuales, para los docentes, son imprescindibles en la enciclopedia del abogado.

Los cursos no profesionalizantes discurren por diferentes géneros prioritariamente académicos, sin excluir algunos profesionales. Mientras que los cursos profesionalizantes solo abordan géneros del ámbito jurídico. También hay una diferencia significativa entre el número de textos que leen y escriben.

Se leen y se escriben más textos en los cursos que no son disciplinares, lo cual resulta paradójico, dado a la importancia que manifiestan los docentes de los cursos disciplinares frente a la lectura y la escritura.

Los egresados de Derecho manifiestan la coincidencia entre algunos de los textos presentes en su formación y los presentes en su actividad profesional. Sin embargo, existen marcadas diferencias entre los textos de los cursos profesionalizantes y los textos de los cursos no profesionalizantes. Los textos del segundo grupo de cursos pierden su relevancia y presencia en el ejercicio profesional. Esto genera nuevas preguntas sobre las prácticas docentes en los campos específicos y las asignaturas de formación. Además, los textos de los cursos profesionalizantes si bien presentan una coincidencia relativa, su grado de coincidencia queda sujeto al campo de desempeño y a lo referido al cargo.

Este ejercicio investigativo permite evidenciar que la pregunta por si en la universidad se debe enseñar primero la lectura crítica o la lectura disciplinar está por resolverse. Pues, al parecer las demandas del medio para los profesionales se han hecho demasiado técnicas y estandarizadas, lo que no permite observar la pertinencia de los cursos de formación y de los desarrollos sobre el ejercicio de la lectura crítica. Por otro lado, esto implica que los abogados en su ejercicio funcionan en roles de tipo técnico, estandarizados, que en muchos casos requieren una aplicación técnica, fundamentada en el conocimiento de las leyes y las jurisprudencias al respecto que actualizan las leyes y generan pautas, guías y antecedentes para posteriores ejercicios de aplicación legal.

Lo anterior pone el énfasis en la especificidad disciplinar que tiene lugar en el ejercicio del Derecho y el rol del profesional de esta disciplina. El profesional del Derecho opera como bisagra entre los ciudadanos, las organizaciones y los marcos legales e institucionales. En este sentido se pueden ver favorecidos el uso de los textos disciplinares en la formación. Pero esta situación no necesariamente pone un dique al uso de textos que no sean disciplinares, pues estos podrían contribuir al ejercicio práctico del relacionamiento con los usuarios que no comparten el tecnolecto, en este caso el lenguaje jurídico especializado.

Finalmente, queda claro que, en el ejercicio profesional cotidiano del grueso de los abogados que participaron en la investigación, se privilegian la interpretación y la aplicación de leyes y de las formas institucionalizadas de los géneros. Es decir que se hace un uso de las leyes y la jurisprudencia en el sentido de aplicar las leyes, de resolver en los marcos de ley y de producir documentos legales.

En esta perspectiva, en la emergencia de los géneros más usados tanto en la formación como en la práctica profesional, se observa que la fuerte presencia de géneros como demandas tutelas, derechos de petición, contestaciones, leyes y sentencias permite señalar cierto privilegio hacia el conocimiento de la ley y la jurisprudencia, sobre posibles ejercicios de pensamiento y lectura crítica.

\section{Reconocimientos}

Este artículo presenta los resultados de la investigación "Identificación de los géneros académicos y profesionales en el ámbito jurídico en Facultad de Ciencias Políticas y Jurídicas de la Institución Universitaria de Envigado", realizada en 2019, bajo el auspicio de la Oficina de Investigaciones de la IUE a través de la modalidad de iniciativa propia. 


\section{Referencias bibliográficas}

Aguilar, P. (2017). Una propuesta de géneros discursivos escritos del ámbito universitario, jurídico y chileno, orientada a la alfabetización académica de estudiantes de derecho. Perfiles Educativos, 39(155), 179-192. Recuperado de http://www. scielo.org.mx/scielo.php?script=sci_arttext\&pi$d=$ S0185-26982017000100179

Albi, A. (2007). Los géneros jurídicos. En E. Alcaraz, J. M. Martínez y F. Yus (coords.), Las lenguas profesionales y académicas (pp. 141-143). Barcelona: Ariel.

Alcaraz, E. y Hughes, B. (2002). Legal translation explained. Manchester: St. Jerome.

Bajtín, M. (1982). Estética de la creación verbal. México: Siglo XXI Editores.

Betancurt, R. y Frías, L. (2015). Competencias argumentativas de los estudiantes de derecho en el marco de las pruebas Saber-Pro. Civilizar, 15(28), 213228. Recuperado de https://revistas.usergioarboleda.edu.co/index.php/ccsh/article/view/289

Bhatia, V. (1999). Integrating products, processes, purposes and participants in professional writing. En C. Candlin y K. Hyland (eds.), Writing: texts, processes and practices (pp. 21-39). Londres: Longman.

Bhatia, V. (2002). Applied genre analysis: a multiperspective model. Ibérica, Revista de la Asociación Europea de Lenguas para Fines Específicos, (4), 3-19. Recuperado de https://www.redalyc.org/ pdf/2870/287026292001.pdf

Bhatia, V. (2008). Lenguas con propósitos específicos: perspectivas cambiantes y nuevos desafíos. Revista Signos, 41(67), 157-176. Recuperado de https:// www.redalyc.org/pdf/1570/157013776004.pdf

Bol, A., Sáiz, M. y Pérez, M. (2013). Validación de una encuesta sobre la actividad docente en Educación Superior. Aula Abierta, 41(2), 45-54. Recuperado de https://riubu.ubu.es/handle/10259.4/2524

Cardona, Á. M., Valencia, E., Duque, J. H. y Londoño, D. A. (2015). Construcción de los planes de vida de los jóvenes: una experiencia de investigación en la vereda La Doctora, Sabaneta (Antioquia). Aletheia, Revista de Desarrollo Humano, Educativo y Social Contemporáneo, 7(2), 90-113.
Recuperado de http://www.scielo.org.co/scielo. php?pid=S2145-03662015000200005\&script=sci_abstract\&tlng=en

Carlino, P. (2005). Escribir, leer y aprender en la universidad. Una introducción a la alfabetización académica. Buenos Aires: Fondo de Cultura Económica.

Cassany, D. (2006). Taller de textos. Leer, escribir y comentar en el aula. Barcelona: Paidós.

Cassany, D. y López, A. (2010). De la universidad al mundo laboral: continuidad y contraste entre las prácticas letradas académicas y profesionales. En G. Parodi (ed), Alfabetización académica y profesional en el siglo XXI: leer y escribir desde las disciplinas (pp. 347-374). Santiago de Chile: Ariel.

Cassany, D. y Morales, O. (2009). Leer y escribir en la universidad: los géneros científicos. En D. Cassany (comp.), Para ser letrados (pp. 109-128). Barcelona: Paidós.

Castañeda, L. y Henao, J. (2005). La lingüística textual y la cultura escrita en la universidad. Zona Próxima, (6), 12-31. Recuperado de https://www.redalyc. org/pdf/853/85300601.pdf

Castelló, M. (2009). Escribir y comunicarse en contextos científicos y académicos: conocimientos y estrategias. Barcelona: Graó.

Castro, M.C., Hernández, L. y Sánchez, M. (2010). El ensayo como género académico: una aproximación a las prácticas de escritura en la universidad pública mexicana. En G. Parodi (ed.), Alfabetización académica y profesional en el siglo XXI: leer y escribir desde las disciplinas (pp. 49-70). Santiago de Chile: Ariel.

DeJarnatt, S. (2001). Law talk: speaking, writing, and entering the discourse of law. Duquesne Law Review, 40, 489. Recuperado de https://heinonline.org/HOL/LandingPage?handle=hein.journals/ duqu40\&div $=24 \& i d=\&$ page $=$

Fuster, Y. (2016). El texto académico como género discursivo y su enseñanza en la educación terciaria. Palabra Clave (La Plata), 5(2), 1-11. Recuperado de https://www.redalyc.org/pdf/3505/350545716002. pdf

García, M. C. y Agüero, C. (2014). Bases para el estudio de la dinámica discursiva en la comunidad jurídica 
chilena. Revista de Derecho (Valdivia), 27(1), 5979. Recuperado de https://scielo.conicyt.cl/scielo. php?pid=S0718-09502014000100003 \&script=sci_arttext\&tlng=e

González, B. y Vega, V. (2010). Prácticas de lectura y escritura en la universidad. El caso de cinco asignaturas de la Universidad Sergio Arboleda. Bogotá: Fondo de Publicaciones Universidad Sergio Arboleda.

Gordillo, A. y Flórez, M. (2017). Los niveles de comprensión lectora: hacia una enunciación investigativa y reflexiva para mejorar la comprensión lectora en estudiantes universitarios. Bogotá: Universidad La Salle.

Henao, J. I., Londoño, D., Frías, L. y Marín, S. (2010). Análisis del discurso en la formación de lectores críticos, autónomos y democráticos. Bucaramanga: El Libro Total.

Herrera, J. D. (2010). La comprensión de lo social horizonte hermenéutico de las ciencias sociales. Manizales: Fundación Centro Internacional de Educación y Desarrollo Humano.

Hyland, K. (2003). Genre-Based Pedagogies: a social response to process. Journal of Second Language Writing, 12, 17-29. Recuperado de https://www.researchgate.net/publication/222546974_Genre-based_pedagogies_A_social_response_to_process

Inglehart, E., Narko, K. y Zimmerman, C. (2003). From cooperative learning to collaborative writing in the legal writing classroom. Legal Writing: Journal of the Legal Writing Institute, 9, 185. Recuperado de https://heinonline.org/HOL/LandingPage?hand$l e=$ hein.journals/jlwriins9\&div=10\&id=\&page $=$

Izquierdo, I. G. (2006). Los géneros y las lenguas de especialidad (I). En E. Alcaraz, J. M. Martínez y F. Yus (coords.), Las lenguas profesionales y académicas (pp. 119-126). Madrid: Ariel.

Kittredge, R. y Lehrberger, J. (eds.) (2015). Sublanguage: studies of language in restricted semantic domains. Berlín: Walter de Gruyter GmbH \& Co. KG.

Kurzon, D. (1997). 'Legal language': varieties, genres, registers, discourses. International Journal of Applied Linguistics, 7(2), 119-139.
Recuperado de https://onlinelibrary.wiley.com/doi/ abs/10.1111/j.1473-4192.1997.tb00111.x

Legarre, S. y Vitetta, M. (2016). La crisis del Ilamado "legal writing" y la renovación de la enseñanza de la escritura en las facultades de derecho argentinas. Academia: Revista sobre Enseñanza del Derecho de Buenos Aires, 14(28), 63-83. Recuperado de https://dialnet.unirioja.es/servlet/ articulo?codigo $=5909220$

Londoño, D. A. y Castañeda, L. S. (2010). La comprensión como método en las ciencias sociales. Revista Virtual Universidad Católica del Norte, 1(31), 227 252. Recuperado de https://revistavirtual.ucn.edu. co/index.php/RevistaUCN/article/view/43

Londoño, D., Ramírez, A. y Garay, K. (2019). Prácticas de literacidad jurídica en el ejercicio docente en dos facultades de Derecho de Antioquia. Revista Nebrija de Lingüística Aplicada a la Enseñanza de Lenguas, 13(26), 51-71. Recuperado de https:// revistas.nebrija.com/revista-linguistica/article/ view/313

López, C. (2002). Aproximación al análisis de los discursos profesionales. Revista Signos, 35(51-52), 195-215. Recuperado de https://scielo.conicyt.cl/ scielo.php?pid=S0718-09342002005100013\&script=sci_arttext

Martínez, C. (2012). El muestreo en investigación cualitativa: principios básicos y algunas controversias. Ciência \& Saúde Coletiva, 17, 613-619. Recuperado de https://www.scielosp.org/article/csc/2012. v17n3/613-619/

Meza, P. (2015). La comunicación del conocimiento en el género Tesis de Lingüística: Comparación entre grados académicos. En G. Parodi y G. Burdile (eds.), Leer y escribir en contextos académicos y profesionales: géneros, corpus y métodos (pp. 67112). Santiago de Chile: Ariel.

Montolío, E. y López, A. (2010). Especificidades discursivas de los textos profesionales frente a los textos académicos: el caso de la recomendación profesional. En G. Parodi (ed.), Alfabetización académica y profesional en el siglo XXI: leer y escribir desde las disciplinas (pp. 215-248). Santiago de Chile: Ariel. 
Nelken, D. (2004). Using the concept of legal culture. Australian Journal of Legal Philosophy, 29, 1-26. Recuperado de https:/www.law.berkeley.edu/files/ Nelken_-_Using_the_Concept_of_Legal_Culture. pdf

Orlando, V. (2014). Sobre prácticas letradas y estudios universitarios. InterCambios. Dilemas y Transiciones de la Educación Superior, 1(2), 68-73. Recuperado de https://ojs.intercambios.cse.udelar.edu.uy/ index.php/ic/article/view/30

Páramo, P. (2018). La investigación en ciencias sociales: técnicas de recolección de la información. Bogotá: Universidad Piloto de Colombia.

Parodi, G. (coord.) (2008). Géneros académicos y géneros profesionales. Accesos discursivos para saber y hacer. Valparaíso: Ediciones Universitarias de Valparaíso.

Parodi, G., Boudon, E. y Julio, C. (2015). El manual de economía: género entre dos mundos disciplinares. En G. Parodi y G. Burdile (eds.), Leer y escribir en contextos académicos y profesionales: géneros, corpus y métodos (pp. 153-186). Santiago de Chile: Ariel.

Parodi, G., Ibáñez, R., Venegas, R. y González, C. (2010). Identificación de géneros académicos y géneros profesionales: principios teóricos y propuesta metodológica. En G. Parodi (ed.), Alfabetización académica y profesional en el siglo XXI: Leer y escribir desde las disciplinas (pp. 249-290). Santiago de Chile: Ariel.

Ríos, J. (2005). Las sentencias judiciales: estudio y análisis sociolingüístico. Tonos, Revista Electrónica de Estudios Filológicos, 9. Recuperado de https://www. um.es/tonosdigital/znum9/corpora/juridicos.htm

Ruiz, U. y Camps, A. (2009). Investigar los géneros discursivos en el proceso educativo. Revista de Psicodidáctica, 14(2), 211-228. Recuperado de https:// www.redalyc.org/pdf/175/17512724004.pdf
Scharager, J. y Reyes, P. (2001). Metodología de la investigación para las ciencias sociales. Santiago de Chile: Pontificia Universidad Católica de Chile.

Schugar, J. T., Schugar, H. y Penny, C. (2011). A nook or a book? Comparing college students' reading comprehension levels, critical reading, and study skills. International Journal of Technology in Teaching \& Learning, 7(2), 174-192. Recuperado de https://www.the-digital-reader.com/wp-content/ uploads/2012/03/IJTTLNook.pdf

Schumacher, S. A. (2006). Learning to write in code the value of using legal writing exercises to teach tax law. Pittsburgh Tax Review, 4, 103. Recuperado de https://heinonline.org/HOL/LandingPage?handle=hein.journals/pittax4\&div=9\&id=\&page=

Schwartz, M. H. (2008). Expert learning for law students. Carolina del Norte: Carolina Academic Press.

Silverman, D. (ed.) (2016). Qualitative research. Londres: Sage.

Swales, J. (1990). Genre analysis: English in academic and research settings. Cambridge: Cambridge University Press.

Tiersma, P. M., Tiersma, P. y Solan, L. (eds.) (2012). The Oxford handbook of language and law. Oxford: Oxford University Press.

Torres, A. (2018). ¿Escritura disciplinar? Una experiencia a partir de la lectura crítica y la habilidad argumentativa en la universidad. Revista Mexicana de Investigación Educativa, 23(76), 95-124. Recuperado de http://www.scielo.org.mx/scielo.php?pi$d=S 1405-66662018000100095 \&$ script=sci_arttext

Wingate, U. (2012). Using academic literacies and genre-based models for academic writing instruction: A'literacy journey. Journal of English for Academic Purposes, 11(1), 26-37. Recuperado de https://www.sciencedirect.com/science/article/pii/ S1475158511000816 